\title{
Attribute Vector Guided Groupwise Registration
}

\author{
Qian Wang ${ }^{1,2}$, Pew-Thian Yap ${ }^{2}$, Guorong $\mathrm{Wu}^{2}$, and Dinggang Shen ${ }^{2, *}$ \\ ${ }^{1}$ Department of Computer Science, University of North Carolina at Chapel Hill \\ qianwang@cs . unc . edu \\ ${ }^{2}$ Department of Radiology and BRIC, University of North Carolina at Chapel Hill \\ \{ptyap, grwu, dgshen\} amed. unc. edu
}

\begin{abstract}
Groupwise registration has been recently introduced for simultaneous registration of a group of images with the goal of constructing an unbiased atlas. To this end, direct application of information-theoretic entropy measures on image intensity has achieved various successes. However, simplistic voxelwise utilization of image intensity often neglects important contextual information, which can be provided by more comprehensive geometric and statistical features. In this paper, we employ attribute vectors, instead of image intensities, to guide groupwise registration. In particular, for each voxel, the attribute vector is computed from its multiple-scale neighborhoods to capture geometric information at different scales. Moreover, the probability density function (PDF) of each attribute in the vector is then estimated from the local neighborhood, providing a statistical summary of the underlying anatomical structure. For the purpose of registration, Jensen-Shannon (JS) divergence is used to measure the PDF dissimilarity of each attribute at corresponding locations of different individual images. By minimizing the overall JS divergence in the whole image space and estimating the deformation field of each image simultaneously, we can eventually register all images and build an unbiased atlas. Experimental results indicate that our method yields better registration quality, compared with a popular groupwise registration method.
\end{abstract}

\section{Introduction}

Image registration has been one of the focuses in medical imaging research for decades. It is a key step for many medical image analysis based applications, since intersubject comparison cannot be performed without first normalizing all images onto a common space. To this end, though a lot of methods have been reported, most of them are, however, pairwise in nature, where a floating image is warped to the space of a fixed image. When pairwise approaches are directly applied to a population of images, one subject needs to be selected in advance as the fixed image, to which all other images can be registered. This manual determination inevitably biases the registration and also subsequent analysis. In light of this, recently proposed groupwise registration algorithms consider the image population as a whole, by simultaneously deforming all individual images impartially, with the final goal of constructing an atlas which describes the population in an unbiased manner [1][2]. Inferences drawn

\footnotetext{
* Corresponding author.
} 
using an unbiased atlas can be expected to be more objective, and reflect more accurately the population characteristics.

Several groupwise registration methods have been proposed in literature. Joshi et al. [3] extends a large deformation diffeomorphic mapping algorithm [4] to work in a groupwise manner for unbiased atlas construction. A congealing framework [5] based groupwise registration scheme is proposed in [6], where intensity based entropy drives a gradient-based stochastic optimizer and pushes each image to the population center simultaneously. This method, which originally works for affine transformation only, is further extended by Balci et al. [7] to incorporate B-Splines to model nonrigid deformation. The objective function, called stack entropy in [6] and [7], measures the compactness of the intensity distribution for a certain voxel location across different subjects. Similar to the popular entropy based metrics used in pairwise registration (e.g., mutual information [8][9]), the current formulation of stack entropy considers image intensity as the only feature, and discards local contextual information that can be provided by the voxel neighborhood. Moreover, each voxel contributes equally to the metric, regardless of its anatomical properties.

In this paper, we propose a novel groupwise registration algorithm, which utilizes multi-scale attribute vectors, rather than only image intensities, as feature descriptors. To better capture the variability of each attribute in the attribute vector, its probability density function (PDF) is further estimated from the neighborhood. Then, the registration algorithm aims to minimize the overall PDF divergence of attribute vectors at each corresponding location across different images. Finally, all the images are groupwisely registered onto a common group mean. Experimental results indicate that our method yields better registration quality and outperforms Balci's method [7].

\section{Method}

Multi-scale attribute vectors are used as voxel anatomical signatures to guide groupwise registration. For a given voxel location, the probability density function (PDF) of each attribute in the vector is estimated from the neighborhood of the voxel. The objective of our groupwise registration is then to minimize the overall PDF divergence of the whole attribute vector, across different subjects, and for each voxel location. The final result is the registration of all images onto a common space defined by the estimated atlas. We will describe our method in more details in the following sections.

\subsection{Attribute Vectors and Their Statistical Descriptions}

In medical image analysis, it is important to build anatomical models that take into account the underlying anatomy, rather than only the similarity of image intensities. Moreover, brain images have intrinsically high anatomical variation, and hence more complex descriptors are needed to fully exploit the structural information. This motivates the utilization of more descriptive features, which take into consideration the different shapes and sizes of anatomical details.

Attribute vectors can capture rich anatomical information, and have been successfully applied to achieve accurate pairwise registration [10]. The composition of the 


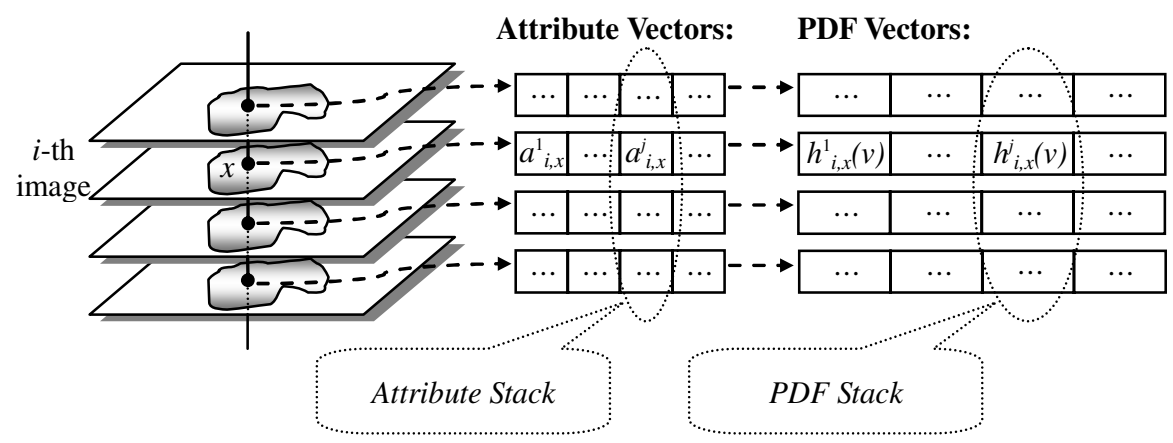

Fig. 1. Attribute vectors at the voxel location. Attribute vectors at the same corresponding location across all subjects form an attribute stack. PDF vectors and PDF stacks are further produced, providing statistical descriptions of attributes in the neighborhood of voxel.

attribute vector is quite flexible and can be easily fine-tuned to cater for different applications. As shown in Fig. 1, we formulate an attribute vector at voxel location $x$ of the ${ }^{i}$-th image as $\vec{A}_{i, x} \triangleq\left\{a_{i, x}^{j} \mid j=1: D\right\}$, where $\boldsymbol{D}$ denotes the number of attributes in the vector. Each attribute $a^{j}{ }_{i, x}$ is a scalar and describes a specific feature, which can constitute the voxel intensity, and also more contextual features such as gradient and directional derivatives. It is, however, worth noting that if the attribute is derived from only a single voxel, the attribute vector can be susceptible to imaging noise and anatomical ambiguity. A better approach is to include attributes which describe contextual information of each voxel by considering its neighborhood. Attributes as such are more robust to imaging noise and can be expected to improve the robustness of the registration. Attributes at the same location across all the subjects then form an attribute stack (as shown in Fig. 1), and we note here that the attribute vectors are always compared stack-wise.

The optimal composition of attribute vectors is related to the specific application. Though complicated features could bring potential benefits to groupwise registration, simple attributes can greatly save computational time, which is critical to groupwise registration where many images need to be handled simultaneously. Therefore, in our study here, only intensities and gradients (calculated in multiple-scale neighborhoods) are used to form the attribute vector, though more complex attributes (i.e., moments [10]) can be incorporated in future. It is demonstrated in the following experiments that sufficiently good performance can be achieved via simple attribute vectors.

To increase the robustness of groupwise registration, a statistical description, in the form of PDF, is estimated for each voxel by considering its neighborhood. And for each attribute vector, we define its corresponding PDF vector. A simple but effective means of estimating PDF $h^{j}{ }_{i, x}(v)$, where $v$ is a random variable, for the attribute $a_{i, x}^{j}$, is by evaluating the histograms of neighboring attribute values around the voxel location $x$. A Gaussian kernel is then used to smooth the histogram. With the PDFs estimated for all attributes in the attribute vector, we can define the PDF vector as $\vec{P}_{i, x} \triangleq\left\{h^{j}{ }_{i, x}(v) \mid j=1: D\right\}$, elements of which now replace roles of scalar attributes $a^{j}{ }_{i, x}$ in the groupwise registration. 


\subsection{Jensen-Shannon Divergence and PDF Stack Divergence}

Jensen-Shannon (JS) divergence [11] is an information-theoretic quantity for measuring the dissimilarity or the distance of two probability distributions. JS divergence was later adopted in [12] for groupwise registration of different point sets. The approach taken was based on the global PDF of each point set. We take a different approach by applying JS divergence on local PDFs, instead on a single global distribution. Noting that the PDF stack for the $j$-th attribute at the location $x$ of $N$ different images is $\left\{h^{j}{ }_{i, x}(v) \mid i=1: N\right\}$, then, the local JS divergence for the PDF stack, or $P D F$ stack divergence, can be written as:

$$
J S^{j}{ }_{x}=\mathcal{H}_{p}\left(\frac{1}{N} \sum_{i=1}^{N} h^{j}{ }_{i, x}(v)\right)-\frac{1}{N} \sum_{i=1}^{N} \mathcal{H}_{p}\left(h^{j}{ }_{i, x}(v)\right)
$$

where $\mathcal{H}_{p}(\cdot)$ is the Shannon entropy with respect to random variable $v$, and each individual image is weighted with equal prior $(1 / N)$. By rewriting $(1)$ as:

$$
J S^{j}{ }_{x}=\frac{1}{N} \sum_{i=1}^{N} \int h^{j}{ }_{i, x}(v) \cdot \log \frac{h^{j}{ }_{i, x}(v)}{\sum_{s=1}^{N} h^{j}{ }_{s, x}(v) / N} d v
$$

we can observe that the local JS divergence for a specific PDF stack measures the variation of the PDFs from the mean PDF, obtained by averaging the PDFs across $N$ different subjects.

\subsection{Overall JS Divergence}

We then formulate the overall JS divergence based on all local PDF stack divergences. It is worth noting that different attributes in the vector are usually orthogonal (e.g. different directional derivatives) or statistically independent (e.g. intensity and gradient). To ensure measurements of local PDF stack divergences at different locations are minimally correlated, the locations for consideration are randomly and sparsely sampled from the image space. Therefore, the overall JS divergence, as the objective function of our groupwise registration algorithm, can be represented by the linear combination of PDF stack divergences of all attributes and all voxel locations in the image volume, that is:

$$
J S_{\text {overall }}=\sum_{x} \sum_{j} w_{j} \cdot J S^{j}
$$

where $w_{j}$ is the empirically determined weighting factor for the $j$-th attribute and complies to $\sum_{j} w_{j}=1$. Suppose $d_{i, x}$ represents the new location transformed from $x$ for the $i$-th image $\left(d_{i, x}=x+\Delta x_{i}\right)$, groupwise registration is achieved by minimizing the overall JS divergence in (3) through a gradient based optimization approach, following:

$$
\frac{\partial J S_{\text {overall }}}{\partial d_{i, x}}=\sum_{x} \sum_{j} w_{j} \cdot \frac{\partial J S_{x}^{j}}{\partial d_{i, x}}
$$


Generally, the optimization framework similar to [7] can be adopted. The B-Spline deformation model is employed in our method to represent the spatial deformation fields $\left\{d_{i, x}\right\}$. The optimizer will simultaneously estimate individual transformations for all images, through minimizing Equation (3). The mean identity transformation constraint is enforced to solve the global drift issue. To avoid local minima, which are quite prone to happen in a high degrees-of-freedom (DOF) optimization problem, a multiresolution strategy is employed. The attribute vectors and their corresponding PDF vectors are computed at different scales. At a coarser level, the optimization problem is effectively reduced to a lower DOF version, and many local minima can be avoided. The registration is progressively refined as the finer resolutions are considered.

\section{Experimental Results}

We have employed the NA0 Database [13] to demonstrate the capability of our method. In the dataset, there are 16 individual brain MR images, each of which has 32 manual anatomical labels. Balci's method [7] is used as a benchmark since it is opensource and is freely available in ITK. Also, it is worth mentioning that our method is implemented by modifying Balci's method in ITK, so that the improvement yielded by the utilization of attribute vectors can be quantified. For fair comparison, we use the same multi-resolution registration strategy (three resolutions) and the same configuration (DOF of transformations and number of iterations) for both Balci's method and our method. After groupwise registration, all aligned images are averaged voxelwise to form the group mean which defines a common space.

To quantify the performance, we calculate the intensity residual errors between 16 aligned images and their group mean. Then, an averaged standard deviation volume for the 16 images is produced. In Fig. 2 (a) and (b), same slices of the residual error volumes are shown at different resolutions for visual comparison. Results yielded by our method are generally darker, especially in areas marked by the ovals, implying lower residual errors. It is worth noting that higher residual errors are more likely to happen near the cortical region, where the inter-subject anatomical variability is generally higher. Lower residual errors usually mean that images are better aligned, and imply higher registration accuracy. The distributions of the residual errors are also shown in Fig. 2 (c). Unsurprisingly, our method yields a distribution with peaks moved closer to zero. In fact, the average of residual errors provided by our method is 3.56\% lower than that of Balci's method. Given that the same number of iterations and similar computation time is used, the improvement brought about by our method in terms of residual errors is attractive.

The ability to establish correspondences of the same anatomical structures from different brains is essential for consistent groupwise registration. We can quantitatively measure this by utilizing the estimated transformation for each image to warp the manually delineated anatomical labels to the common space, and then compare the overlap rates of these labels. The overlap rate utilized here is the Jaccard coefficient, which is defined as the ratio between the size of the region of intersection to the size of the regional union of a registered image and the mean image. In Fig. 3, we provide the average overlap rates for all 32 labels, produced via Balci's method and our method, respectively. Our method shows improvement in all 32 labels, with an average of $2.45 \%$ increase, implying that more consistent correspondence matching can be achieved using our method. 


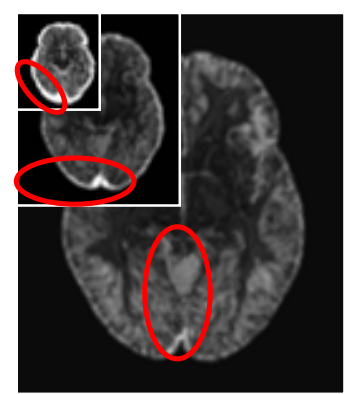

(a)

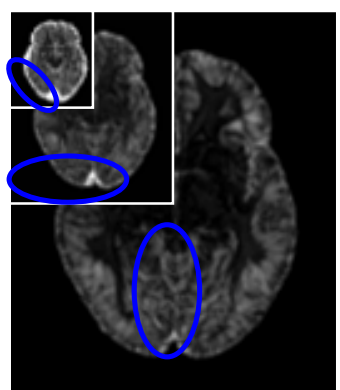

(b)

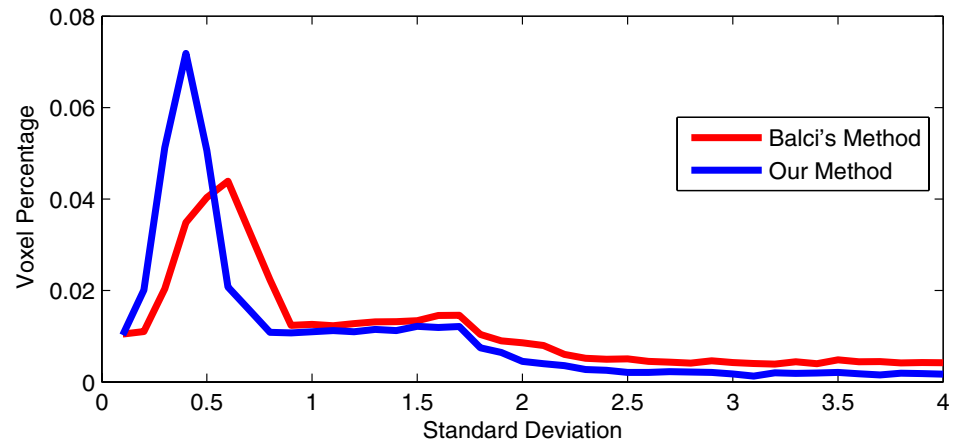

(c)

Fig. 2. Comparison of residual errors after groupwise registration via two different methods. (a) and (b): Our method (blue) produces lower errors than Balci's method (red), (c): The distributions of residual errors yielded by the two methods.

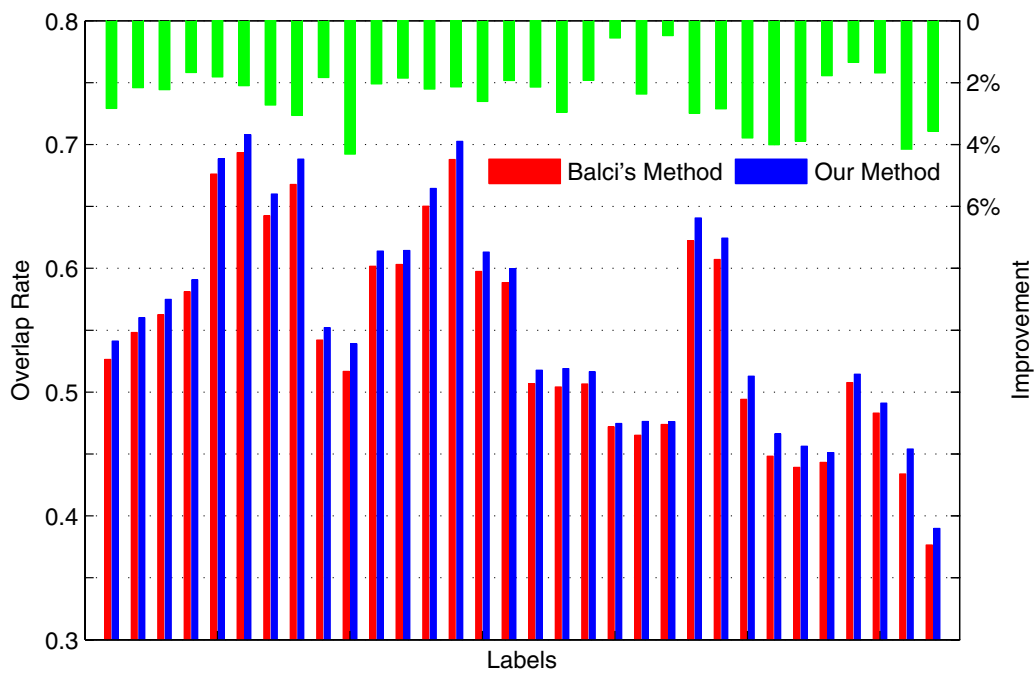

Fig. 3. Our method produces higher overlap rates than Balci's method in all 32 labels. The green bars represent the percentage of improvement by our method, relative to Balci's method. 


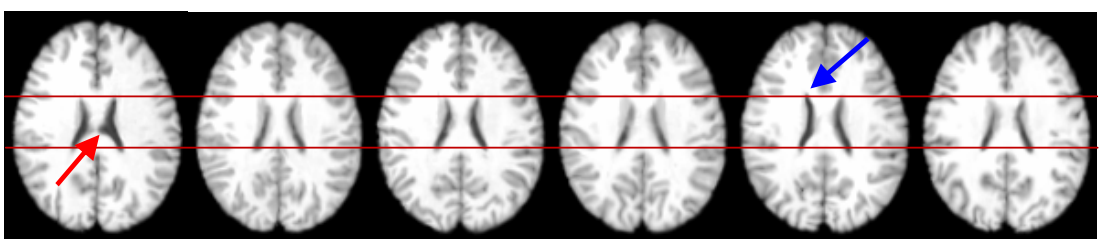

(a)



(b)

Fig. 4. Corresponding slices are selected from the images registered via Balci's method (a) and our method (b), respectively. Ventricles in row (b) are more similar to each other than those in row (a), especially for the locations indicated by arrows.

Furthermore, our method could better align anatomical structures in groupwise registration. For example, as shown in Fig. 4, we provide the same slices of 6 images selected from previously registered 16 images. The rows of (a) and (b) in Fig. 4 show the results produced via Balci's method and our method, respectively. As indicated by arrows, images registered by our method are much consistent than those yielded by Balci's method. In particular, ventricles in row (b) are more similar to each other than those in row (a).

\section{Conclusion}

In this paper, we have presented a novel groupwise registration method. Our method employs attribute vectors as anatomical signatures of voxels. We then estimate the PDF of each attribute at a certain voxel location from its neighborhood. Across different images, the dissimilarity of the PDF stack is further measured by the JS divergence. We can finally achieve groupwise registration by minimizing the overall JS divergence in a multi-resolution manner. Experimental results indicate that our method can yield better registration results than Balci's method, using the same implementation freely available in ITK.

Acknowledgments. This work is supported by NIH grants 1R01EB006733 and 1R01EB009634.

\section{References}

1. Zitová, B., Flusser, J.: Image Registration Methods: A Survey. Image and Vision Computing 21, 977-1000 (2003)

2. Crum, W.R., Hartkens, T., Hill, D.L.G.: Non-rigid Image Registration: Theory and Practice. The British Journal of Radiology 77, S140-S153 (2004) 
3. Joshi, S., Davis, B., Jomier, M., Gerig, G.: Unbiased Diffeomorphic Atlas Construction for Computational Anatomy. NeruoImage 23, S151-S160 (2004)

4. Miller, M.I., Younes, L.: Group Actions, Homeomorphisms, and Matching: A General Framework. International Journal of Computer Vision 41, 61-84 (2001)

5. Learned-Miller, E.G.: Data Driven Image Models through Continuous Joint Alignment. IEEE Trans. Pattern Analysis and Machine Intelligence 28, 236-250 (2006)

6. Zöllei, L., Learned-Miller, E., Grimson, E., Wells, W.: Efficient Population Registration of 3D Data. In: Liu, Y., Jiang, T.-Z., Zhang, C. (eds.) CVBIA 2005. LNCS, vol. 3765, pp. 291-301. Springer, Heidelberg (2005)

7. Balci, S.K., Golland, P., Wells, W.M.: Non-rigid Groupwise Registration using B-Spline Deformation Model. In: Workshop on Open-Source and Open-Data for 10th MICCAI, pp. 105-121 (2007)

8. Maes, F., Collignon, A., Vandermeulen, D., Marchal, G., Suetens, P.: Multi-modality Image Registration by Maximization of Mutual Information. IEEE Trans. Medical Imaging 16, 187-198 (1997)

9. Wells, W.M., Viola, P., Atsumi, H., Nakajima, S., Kikinis, R.: Multi-modal Volume Registration by Maximization of Mutual Information. Medical Image Analysis 1, 35-51 (1996)

10. Shen, D., Davatzikos, C.: HAMMER: Hierarchical Attribute Matching Mechanism for Elastic Registration. IEEE Trans. Medical Imaging 21, 1421-1439 (2002)

11. Lin, J.: Divergence Measures Based on the Shannon Entropy. IEEE Trans. Information Theory 37, 145-151 (1991)

12. Wang, F., Vemuri, B.C., Rangarajan, A., Eisenschenk, S.J.: Simultaneous Nonrigid Registration of Multiple Point-Sets and Atlas Construction. IEEE Trans. Pattern Analysis and Machine Intelligence 30, 2011-2022 (2008)

13. Christensen, G.E., Geng, X., Kuhl, J.G., Bruss, J., Grabowski, T.J., Pirwani, I.A., Vannier, M.W., Allen, J.S., Damasio, H.: Introduction to the Non-rigid Image Registration Evaluation Project (NIREP). In: Pluim, J.P.W., Likar, B., Gerritsen, F.A. (eds.) WBIR 2006. LNCS, vol. 4057, pp. 128-135. Springer, Heidelberg (2006) 